\title{
Dietary phenolics other than anthocyanins inhibit PTP1B; an in vitro and in silico validation
}

\author{
S.K. Barik ${ }^{1}$, W.R. Russell ${ }^{1}$, B. Dehury ${ }^{2}$, M. Cruickshank ${ }^{1}$, K.M. Moar ${ }^{1}$, D. Thapa ${ }^{1}$ and \\ N. Hoggard ${ }^{1}$ \\ ${ }^{1}$ The Rowett Institute, University of Aberdeen, Aberdeen, AB25 2ZD, United Kingdom and \\ ${ }^{2}$ RMRC, Indian Council of Medical Research, Bhubaneswar, 751023, Odisha, India
}

Previous reports suggest that berries rich in dietary anthocyanins are able to inhibit protein tyrosine phosphatase (PTP1B), an important target in the prevention of type 2 diabetes $^{(1,2)}$. Black and green currants (Ribes sp.) were selected due to their significant difference in anthocyanin content ${ }^{(3)}$, which allowed us to assess if the metaboloites produced following in vitro gastrointestinal digestion (IVGD) are responsible for PTP1B inhibition.

Freeze-dried black and green currant extracts underwent IVDG $^{(4)}$. The predominant metabolites produced were identified by LC-MS/MS and these and the digested extracts were screened for their ability to inhibit human PTP1B in vitro ${ }^{(5)}$. For the most effective inhibitor, Lineweaver-Burk and Dixon plots were employed at mean reciprocal of initial velocity for $n=3$ replicates at several substrate concentrations. Further, targeted molecular docking was used to study the ligand-protein interaction of the same compound using AutoDock Vina V4.2(6).

IVGD black and green currant extracts at a physiological relevant concentration inhibited PTP1B by $60.2 \%(\mathrm{P}<0.001)$, IC50 $11.42 \pm 0.16 \mu \mathrm{g} / \mathrm{mL}$ and $45.7 \%(\mathrm{P}<0.001)$, IC50 $19.98 \pm 0.21 \mu \mathrm{g} / \mathrm{mL}$ respectively. This suggests that anthocyanins may not be the key inhibitors of PTP1B. Phytochemical analysis identified several predominant phenolics, which were further studied to identify their individual ability to inhibit PTP1B. Gallic acid at a physiological relevant concentration inhibited PTP1B by 52.9\% $(\mathrm{P}<$ 0.001 , IC50 $56.37 \pm 1.03 \mu \mathrm{g} / \mathrm{mL})$, followed by 4-hydroxybenzaldehyde $(38.9 \%, \mathrm{P}>0.001)$, ferulic acid $(37.7 \%, \mathrm{P}<0.001)$ resveratrol $(37.3 \%, \mathrm{P}<0.001)$ and chlorogenic acid $(36.1 \%, \mathrm{P}<0.001)$. Sodium orthovanadate (positive inhibitor) at the same concentration inhibited PTP1B by $56.5 \%(P=0.0034$, IC50 $4.65 \pm 0.80 \mu \mathrm{g} / \mathrm{mL})$. Co-incubation of the phenolics did not increase PTP1B inhibition when compared to their individual compounds suggesting a non-synergistic effect. Phytochemical analysis identified that gallic acid was the most abundant phenolic (14.9\%) compound measured in green currants. Moreover, enzyme kinetics presented that gallic acid inhibits PTP1B non-competitively $(\mathrm{Ki}=33.3 \pm 1.67 \mu \mathrm{g} / \mathrm{mL})$ while the in silico study identified the binding affinity of gallic acid to PTP1B's active site to be $-6.37 \mathrm{kcal} / \mathrm{mole}$. In conclusion, we have shown that several phenolic compounds from the currants are able to inhibit human PTP1B in vitro with gallic acid having the highest activity.

The University of Aberdeen and Nutricia Research Foundation funded the doctoral studentship to SKB. The research was supported by the Rural Affairs Food and the Environment Strategic Research (RESAS). Authors are thankful to the James Hutton Institute, Dundee for providing the currants and Dr Graham Horgan (BioSS) for his assistance during statistical analysis of the study.

1. Xiao T, Guo Z, Sun B et al. (2017) J Agric Food Chem 65, 6211-6221.

2. Zhao BT, Duc Hung Nguyen, Le DD, et al. (2018) Arch Pharm Res 41, 130-161.

3. Mattila PH, Hellstrom J, Karhu S, et al. (2016) Food Chem 1, 14-20.

4. Minekus M, Alminger M, Alvito P, et al. (2014) Food Funct 5, 1113-1124.

5. Uddin MN, Sharma G, Yang J, et al. (2014) Phytochemistry 103, 99-106.

6. Dehury B, Behera SK, Mahapatra N (2017) J Mol Graph 71, 154-166. 